\title{
Norois
}

Environnement, aménagement, société

\section{Le mythe du paysage qui vote}

Géographies, héritages socio-culturels et tendances électorales contemporaines dans les Deux-Sèvres

The myth of the landscape that votes. Geographies, socio-cultural heritages and contemporary electoral trends in Deux-Sèvres

\section{Nicolas Gamache}

\section{CpenEdition}

\section{Journals}

Édition électronique

URL : http://journals.openedition.org/norois/594

DOI : $10.4000 /$ norois. 594

ISBN : 978-2-7535-1542-0

ISSN : $1760-8546$

\section{Éditeur}

Presses universitaires de Rennes

\section{Édition imprimée}

Date de publication : 1 mars 2005

Pagination : 7-26

ISBN : 978-2-7535-0100-3

ISSN : 0029-182X

Référence électronique

Nicolas Gamache, «Le mythe du paysage qui vote », Norois [En ligne], 194 | 2005/1, mis en ligne le 12 août 2008, consulté le 19 avril 2019. URL : http://journals.openedition.org/norois/594 ; DOI : 10.4000/ norois.594

Ce document a été généré automatiquement le 19 avril 2019

(c) Tous droits réservés 


\title{
Le mythe du paysage qui vote
}

\author{
Géographies, héritages socio-culturels et tendances électorales \\ contemporaines dans les Deux-Sèvres \\ The myth of the landscape that votes. Geographies, socio-cultural heritages and \\ contemporary electoral trends in Deux-Sèvres
}

\section{Nicolas Gamache}

\section{NOTE DE L'ÉDITEUR}

Cet article a été reçu le 29 mai 2004 et définitivement accepté le $1^{\text {er }}$ février 2005.

1 Le département des Deux-sèvres (fig. 1) en Poitou-Charentes, tient son nom des deux cours d'eau prenant leur sur les hauteurs de Gâtine et du plateau Mellois : la Sèvre nantaise et la Sèvre niortaise. C'est peut-être là le seul lien entre le nord et le sud de ce territoire. Rien ne laissait présager jusqu'à la constituante en 1790 (Merle, 1990), la formation d'un département à partir de cet espace tout de long au cœur de l'ancienne province du Poitou, entre aujourd'hui Vienne et Vendée. Tout sépare dans la "géographie » un nord aux marges du massif armoricain, sur socle de granite et de schistes aux paysages de bocage, d'un sud sur terrains calcaires aux paysages le plus souvent ouverts, ou encore marécageux comme au marais poitevin. Cette dichotomie physico-spatiale des Deux-Sèvres a laissé place à un imaginaire qui perdure et franchit les générations: les façons de penser, de voir le monde et de voter dépendraient des conditions du milieu. A. Siegfried en 1913 rapportait le dicton populaire vendéen énonçant « le calcaire produit l'instituteur, le granit produit le curé ». Ce dicton encore répandu explique ainsi « que le calcaire vote à gauche (Thouarsais, Niortais et sud DeuxSèvres) et le granite vote à droite (Bressuirais et Gâtine) ». Nous nous proposons d'explorer cette croyance, à la lumière notamment des travaux de M. Bussi (1993) : sols et paysages, religion et héritages socio-culturels seront mobilisés. Nous nous engagerons ensuite dans la voie des facteurs socio-démographiques, par les mobilités et recompositions socio-démographiques des populations. L'intérêt de cette présentation 
tiendra évidemment à rejeter cette hypothèse d'un déterminisme naturel sur l'expression politique lors des élections. Si les conditions du milieu peuvent influer sur l'exploitation de ce dernier et donc sur la manière de vivre le rapport au monde, elles sont surtout l'expression des rapports sociaux, plus ou moins hiérarchisés, au sein d'une communauté. Sur le temps long, elles aident à comprendre cette relation non causale entre le vote et le milieu. Nous appuierons l'idée du pouvoir du territoire à porter des valeurs (du vote) dans la durée, notamment quant à son caractère symbolique. Les cartes mentales des électeurs par exemple révèlent que ces derniers ont, pour certains, conscience que leur acte s'inscrit dans un lieu chargé d'histoire ou de traditions (Guillorel, 1991). Un territoire de proximité en fin d'article l'illustrera.

Figure 1 : Situation et cantons des Deux-Sèvres

Location and districts of Deux-Sèvres

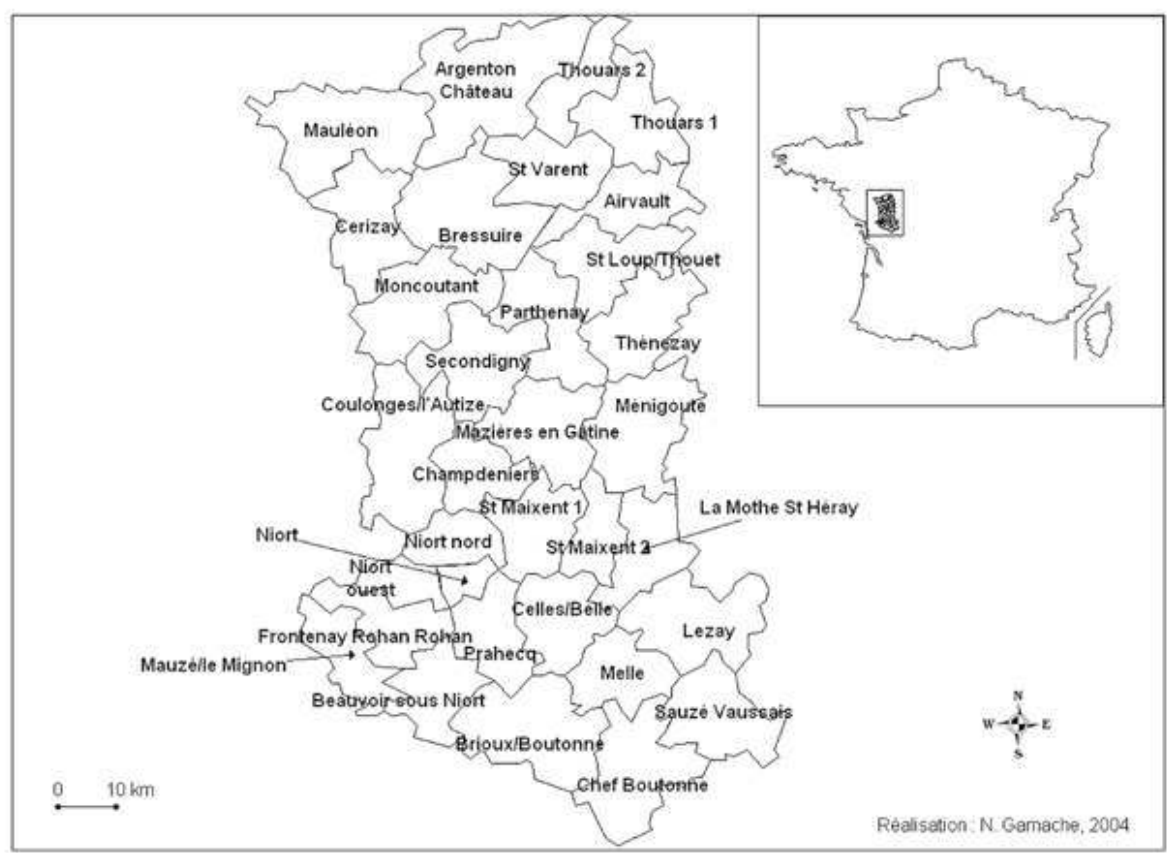

\section{Paysage politique traditionnel}

\section{Géographie, vote et territoire}

La géographie française doit beaucoup à $\mathrm{P}$. Vidal de La Blache (1994), qui au début du $\mathrm{xx}^{\mathrm{e}}$ siècle peignit un Tableau de la géographie de la France et engagea la discipline dans une exploration des liens entre paysages de la France et mentalités de ses habitants. A. Siegfried (1913) s'est largement inspiré de P. Vidal de La Blache pour à son tour jeter un regard sur cette relation dans une perspective politique. En peignant un Tableau politique de la France de l'Ouest, il tente de mettre en lumière ce qui est significatif dans les relations d'un territoire et de ses habitants pour comprendre la permanence des comportements électoraux, dans le temps long notamment. Les facteurs physiques d'une "région naturelle " façonneraient les "genres de vie ", rendant compte de la répartition des opinions politiques. Y. Lacoste (1986), en s'affranchissant de tout déterminisme physique, 
conçoit aussi le territoire comme porteur du vote. Mais c'est la répartition territoriale qui proposerait des systèmes explicatifs et non le territoire en lui-même.

3 Quoi qu'il en soit, le territoire, "domaine socialisé qui se définit en fonction du mode d'organisation socio-politique et du mode de régulation sociale» (Scheibling, 1994) se trouve donc intimement lié au « vote », expression de ce mode d'organisation.

4 Le département des Deux-Sèvres se situe au carrefour des bassins aquitain et parisien et des Massifs Armoricain et Central. Les influences sont donc nombreuses, qu'elles soient physiques ou humaines. Il s'offre comme un cadre d'analyse original dans cette optique.

\section{Cadre physique des Deux-Sèvres}

5 L'historien L. Merle (1990) a dressé un portrait des Deux-Sèvres concis et pertinent: «Rien n'est aussi dissemblable de la région de Gâtine, avec ses fermes isolées, ses champs clos, ses nombreux ruisseaux et ses pâturages verdoyants, que la plaine de Niort, nue, sèche, aux horizons étendus et ses agglomérations rassemblées autour de ses sources clairsemées. Le contraste est encore plus marqué lorsqu'on aborde le Marais, avec ses routes d'eau et ses cultures singulières. "

On pourrait découper le département à partir de nombreuses grilles d'interprétation du milieu étudié: des critères pédologiques, géologiques, paysagers, agronomiques, climatiques, permettraient de multiplier la constitution d'unités physiques différentes. En simplifiant, la mosaïque physique du territoire contient sept types d'entités (fig. 2) : les bocages au nord (Bressuirais, Gâtine, l'Entre Plaine et Gâtine et les Contreforts de la Gâtine) sur les marges du Massif Armoricain, les plaines (de Niort et du Mellois dans la partie sud, de Thouars au nord-est), puis le marais poitevin, la forêt de Chizé au sud, le bocage sur calcaires du Mellois, un vignoble à l'extrême nord du département et enfin les zones urbaines (Thouars, Parthenay, Bressuire, Saint-Maixent-l'École et Niort). 
Figure 2 : Cadre physico-spatial des Deux-Sèvres et pratiques religieuses Surroundings and religious practices in Deux-Sèvres

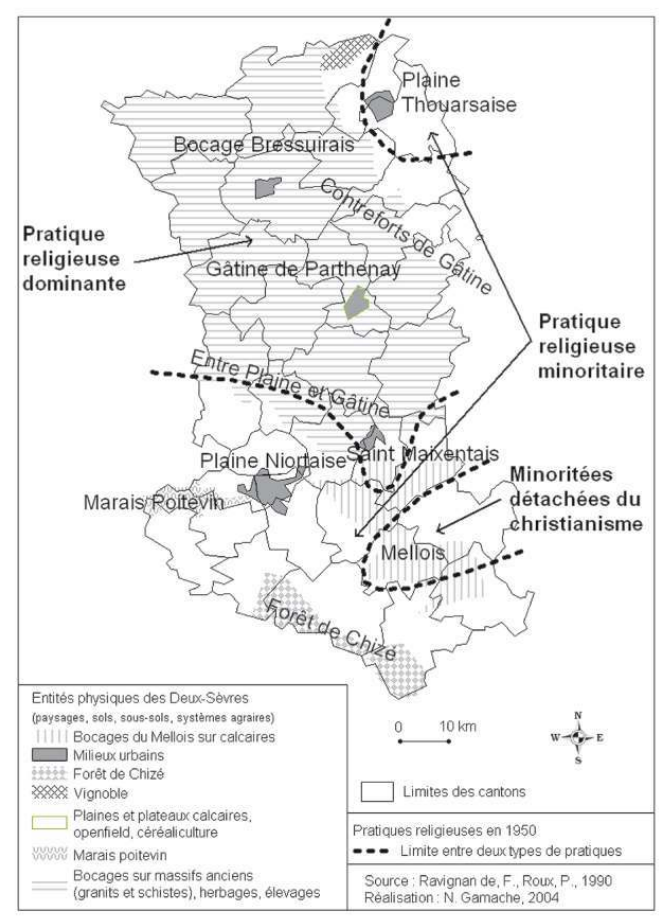

\section{La formation des Deux-Sèvres : un territoire d'abord administratif}

La diversité géographique physique que nous venons d'évoquer démontre que l'homogénéité paysagère et culturelle ne fut pas nécessairement, ni même essentiellement, une préoccupation du législateur lorsque les départements furent créés, au lendemain de la Révolution. L. Merle (1990) souligne dans son ouvrage sur la formation des Deux-Sèvres le but premier de la constitution des départements après la révolution française : «Effacer et faire oublier les institutions de l'Ancien Régime.» Concernant les Deux-Sèvres, le « résultat d'un découpage arbitraire effectué sans le moindre souci des servitudes historiques et géographiques " aboutit à un département ne présentant « aucune unité ».

Mais l'ancienne province du Poitou elle-même (composée des départements actuels de Vendée, Vienne et Deux-Sèvres) recelait ces «deux tendances opposées, pièces ou morceaux sans affinités naturelles » (plaines et bocages) (Merle, 1990).

Les Deux-Sèvres doivent donc leur existence essentiellement à la division du Poitou par les Constituants en trois et non deux départements. «L'Assemblée Nationale se persuada que pour faire disparaître les inégalités de l'Ancien Régime, il était nécessaire d'en briser les cadres territoriaux et de jeter sur la France entière le manteau gris de l'égalité que venait de tailler son Comité de Constitution. » La principale préoccupation tenait en fait à faciliter le déplacement des électeurs vers le chef lieu de circonscription, aux dépends de toute autre considération de commodité administrative. Finalement, au lieu d'avoir deux départements homogènes politiquement issus du Poitou, trois départements ont été créés 
avec aujourd'hui une pluralité politique nord - sud toujours de mise (au moins en DeuxSèvres).

\section{Histoire, religion et structure sociale : le poids des héritages} sur les sociétés où la pratique est élevée ou non, sur certains paramètres territoriaux, l'appropriation, réelle ou symbolique des lieux, et le système de relations sociales. On ne peut s'affranchir d'observer ses évolutions et les mettre en parallèle avec les tendances politiques d'un territoire. On ne s'étendra pas ici plus largement sur ce point, la figure 2 permettant de s'interroger sur la corrélation ou non de la religion à la territorialité et par extension au vote (se rapporter aux travaux de J.-R. Bertrand et C. Muller, 1999).

11 Ce questionnement n'est pas nouveau, on retrouve dans l'historiographie locale cette relation entre le fait religieux et la couleur politique. R. Bobin (1926) écrivait que «la population gâtinelle se rattache à la grande famille celte qui peuple tout l'ouest de la France. Mais la population est mieux définie encore par sa couleur politique : ce sont les chouans. La Gâtine et le Bocage ont été, au temps de la Révolution, le théâtre de la sauvage guerre de Vendée. Aujourd'hui, c'est l'un des quelques pays réactionnaires de la France et c'est avant tout une contrée essentiellement catholique, où l'on rencontre à chaque instant des calvaires, où, le dimanche, l'église s'emplit de fidèles. Rien de tout cela dans la Plaine, républicaine. Si le granite est réactionnaire, par contre, le calcaire est républicain ».

(oriquement, le catholicisme fut donc tout puissant dans le nord Deux-Sèvres et assimilé à la structuration sociale hiérarchisée, s'appuyant sur des valeurs spirituelles pour le respect de l'ordre établi. Ce dernier est surtout celui du rapport entre fermiers et propriétaires, qui conditionne aussi le rapport politique des paysans et la territorialité du vote de l'époque: "Pour conserver la ferme que lui loue quelque noble vendéen, le fermier est obligé quelquefois d'avoir une opinion politique définie et que, pour éviter des ennuis dans son fermage, il vote quelquefois par ordre et par tête » (Bobin, 1926).

13 En 1946, J.-R. Colle va dans le même sens, laissant percevoir une lourdeur des héritages socio-culturels très prégnants dans la vie politique du territoire: «Au point de vue politique, le Gâtinais est plutôt de droite par méfiance des nouveautés, pour plaire à son patron s'il est métayer d'une famille noble, pour des raisons religieuses et surtout par tradition. "

14 Mais si aujourd'hui l'appartenance religieuse, patrimoniale ou de classe, s'estompe, l'appartenance « au sol » résiste (Bussi et Freire-Diaz, 2002) par l'émergence de l'électeur - acteur maitrisant son capital spatial, induisant les réorganisations spatiales des comportements électoraux : vote - pro européen - en grandes villes par exemple.

\section{Territoire et vote : des corrélations, mais pas de déterminisme géographique}

15 Tous les indicateurs qualifiant les Deux-Sèvres jusque-là permettent de "dichotomiser » le territoire deux-sèvriens : un Nord aux bocages sur granite, «finistère » des paysages bretons et des us religieux de cet Ouest français ; un Sud des plaines et plateaux calcaires où la religion est reléguée au second plan lorsqu'elle n'est pas protestante. À ce stade, 
tout soutient l'idée d'un déterminisme entre paysage, culture et vote. Mais la superposition de divers éléments, pour autant que cette superposition soit avérée par les calculs des coefficients de ressemblance spatiale (de Minnick) ou d'association (de Jaccard) (Badariotti, 2004), informe davantage sur des caractères territoriaux qu'elle n'explique les corrélations, toute relative soient-elles, avec les votes.

\section{Évolution socio-économique}

\section{Méthode}

Afin de connaître la "forme de prégnance territoriale du vote ", nous avons cherché les permanences électorales spatiales et les différentiations territoriales du vote. Nous avons choisi pour corpus électoral les élections présidentielles de 2002 et les élections régionales de 2004 par canton. Le rapport gauche/droite met en valeur les permanences. Les facteurs sociaux sont mobilisés pour corréler ou non les votes socialement dans le clivage politique droite/gauche et surtout leur répartition territoriale. Nous avons donc utilisé à partir de la base de données des résultats (du ministère de l'Intérieur) les méthodes d'ajustement et de corrélation (Bussi, 1993). L'étude de cas sur le Ménigoutais en dernière partie, développera quant à elle, des facteurs explicatifs de la relation du vote au territoire à une micro-échelle, celle du canton. Cette démarche se rapproche davantage de celle d'une géographie culturelle électorale. Nous espérons ainsi apporter une contribution significative à une problématique du vote: le territoire, clé de compréhension des permanences électorales?

\section{Une dichotomie spatiale}

17 Trois indicateurs majeurs permettent de dresser un tableau électoral des Deux-Sèvres: l'indice de concentration (Bussi et al., 2004) pour connaître la répartition territoriale des forces de chaque camp, le ratio mettant en rapport chacun de ces camps pour connaître le rapport de force entre ceux-ci, et l'indice d'évolution (Bussi et al., 2004) pour souligner les évolutions et tendances.

\section{Répartition}

On peut aborder la répartition d'un électorat de manière relative (ce que l'on rangera davantage dans le rapport de force puisqu'en l'occurrence le clivage en Deux-Sèvres est essentiellement bipolaire, le cas du FN sera abordé plus loin) ou en données absolues.

La figure 3 donne une idée de la répartition des votes de chaque camp aux élections présidentielles de 2002 et régionales de 2004. L'électorat CPNT a été rattaché dans les calculs à la droite gouvernementale, en raison de la nature de son électorat local. Ces cartes se rapprochent du calcul du « champ de force » ou " champ géographique » (Bussi, 1993). Cette méthode expérimentale rend compte de la spatialité de l'électorat de chacun des partis et donne une lecture simple de la configuration de la distribution des électorats : de même que Bobin l'avait noté en 1926, aujourd'hui encore, la droite tire l'essentiel de ses voix en Bressuirais et en Gâtine, la gauche en Mellois, Niortais et Thouarsais. La figure 4 illustre cette répartition en opposant ces deux principaux clivages. La droite d'ajustement linéaire, outre de qualifier la répartition des votes, permet aussi de 
comparer cette répartition avec celle de l'autre camp. Les indices de concentrations (tableau 1), confirment ces tendances illustrées par les cartes et se calquent même sur celles des taux.

Figure 3 : Répartition des votes de droite, de gauche et FN en Deux-Sèvres aux élections régionales 2004 et présidentielles 2002

Distribution of right, left and FN votes in Deux-Sèvres for the 2004 regional and the 2002 presidential elections

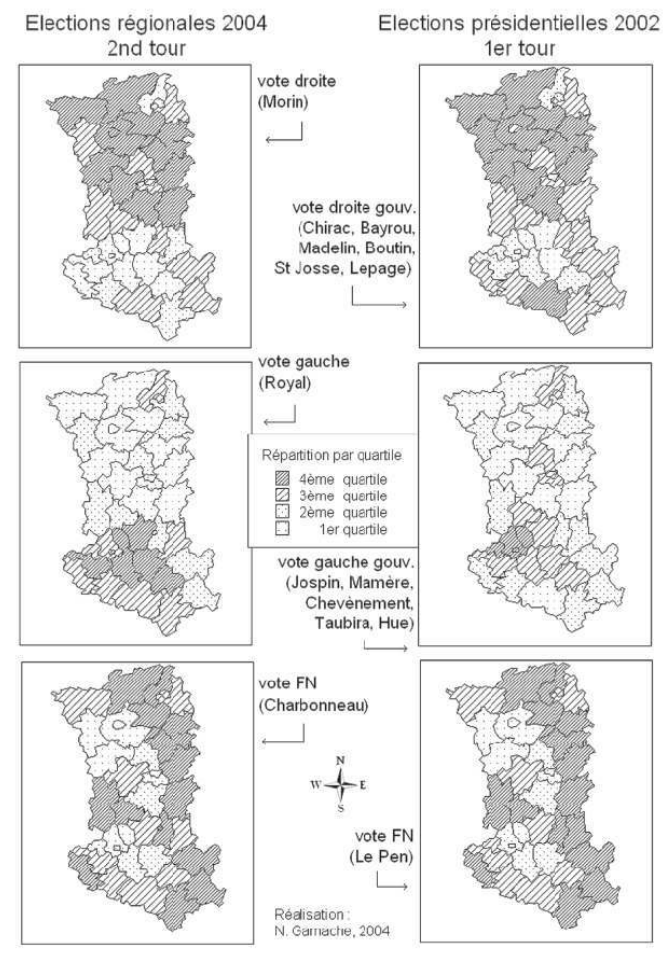

\section{Rapport de forces}

La répartition des forces de chaque camp montre des inégalités sur l'ensemble du département. De même, à l'intérieur de chacun de ces camps et des cantons qui leur sont acquis, des inégalités sont aussi présentes. Ces inégalités sont le fruit de plusieurs facteurs : la présence des votes protestataires («écolos » ou extrêmes), ou encore selon l'échelonnement de gradients entre périphérie et centre de chacun des électorats. La notabilité des candidats, à Celles sur Belle pour Ségolène Royal aux régionales par exemple, marque une adhésion plus forte au cœur de ce système. Ségolène Royal a en effet assumé divers mandats locaux (élue à l'Assemblée nationale en 1988, réélue en 1993, 1997 et 2002, élue au Conseil municipal de Melle de 1989 à 1995, puis de Niort de 1995 à 2001, au conseil général des Deux-Sèvres de 1995 à 2001 et au conseil régional en 1992) ce qui était un avantage relatif sur Élisabeth Morin, originaire de la Vienne, département voisin. La figure 5, en rapportant le poids électoral de la gauche à celui de la droite aux dernières régionales, renvoie l'image d'un rapport de force entre les deux tendances. Elle illustre pour le premier tour des élections régionales les tendances lourdes sur la répartition des cantons entre les votes de droite (Morin pour l'UMP, Fontenay pour le CNPT) et de gauche (Royal pour le PS et la Gauche Unie). Bien qu'un certain nombre de cantons de la partie nord ait un ratio des votes de gauche supérieur aux votes de droite, 
cette dernière marque sa «territorialité » dans le contexte du score régional largement acquis à Ségolène Royal.

\section{Évolutions et tendances}

21 Lorsqu'une élection se caractérise par une nette domination d'un camp (droite ou gauche), celui qui voit ses scores se replier découvre la marginalité de son score dans les fiefs de l'adversaire, tandis que ses rares bastions représentent, plus que jamais, son principal apport de voix. Le nombre de cantons majoritairement acquis à la gauche varie sensiblement d'une élection à l'autre (entre 2002 et 2004, il augmente fortement), mais la géographie de l'implantation de la gauche demeure stable.

La droite d'ajustement de la figure 4 confirme cette dichotomie spatiale avec une corrélation très forte, lors du second tour (la différence étant liée à la présence d'un candidat Front National). L'intérêt ne réside pas dans la corrélation, mais dans la répartition des cantons sur la droite d'ajustement: coupée en deux, on retrouve d'une part la partie nord Deux-Sèvres, d'autre part la partie sud (dans laquelle il faut inclure la plaine Thouarsaise).

Figure 4 : Régression linéaire de la répartition des votes au 2 nd tour des régionales entre Morin et Royal par canton

Linear regression of the votes distribution at the 2 nd round of the regional election between Morin and Royal by district

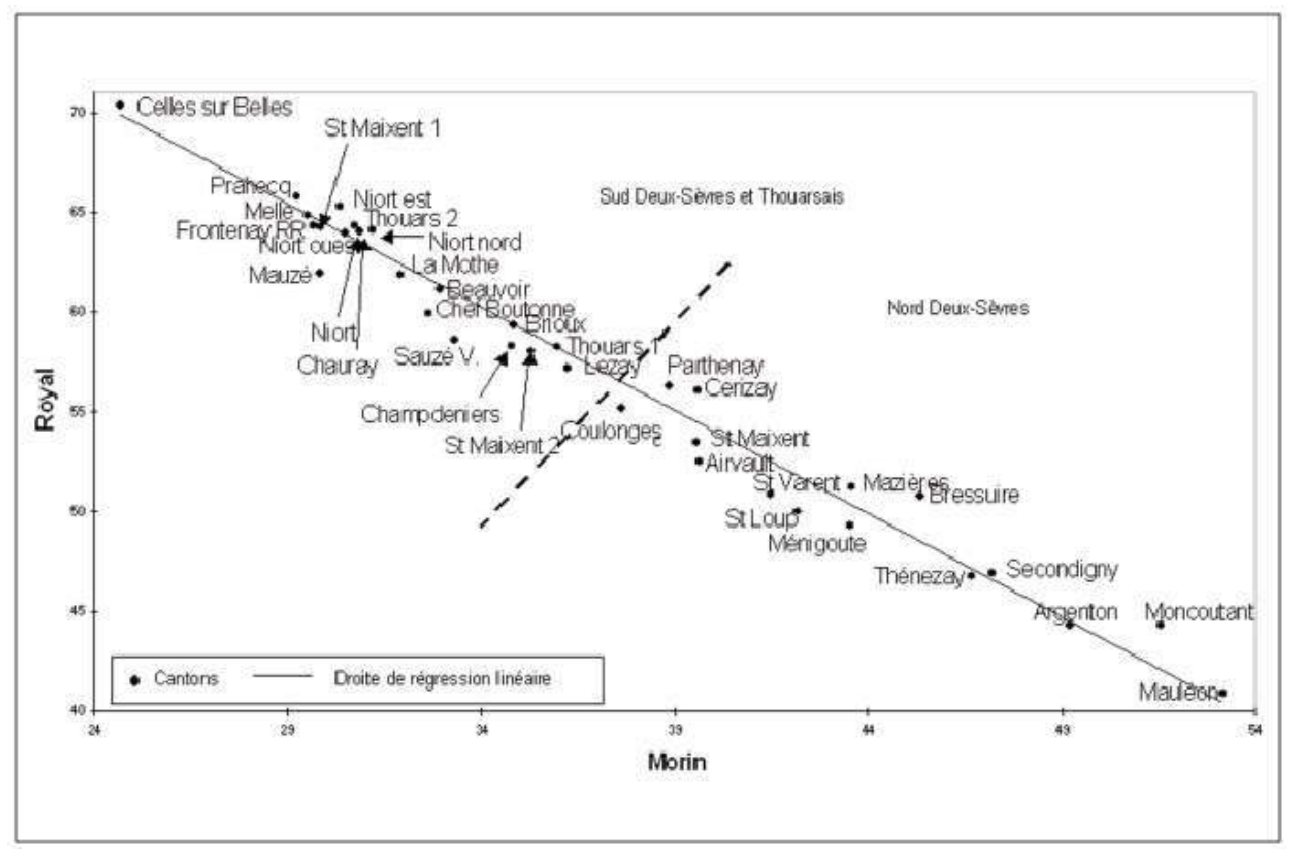


Figure 5 : Rapport de force gauche/droite au premier tour des élections régionales 2004 Balance of power between right and left at the 1st round of the 2004 regional election

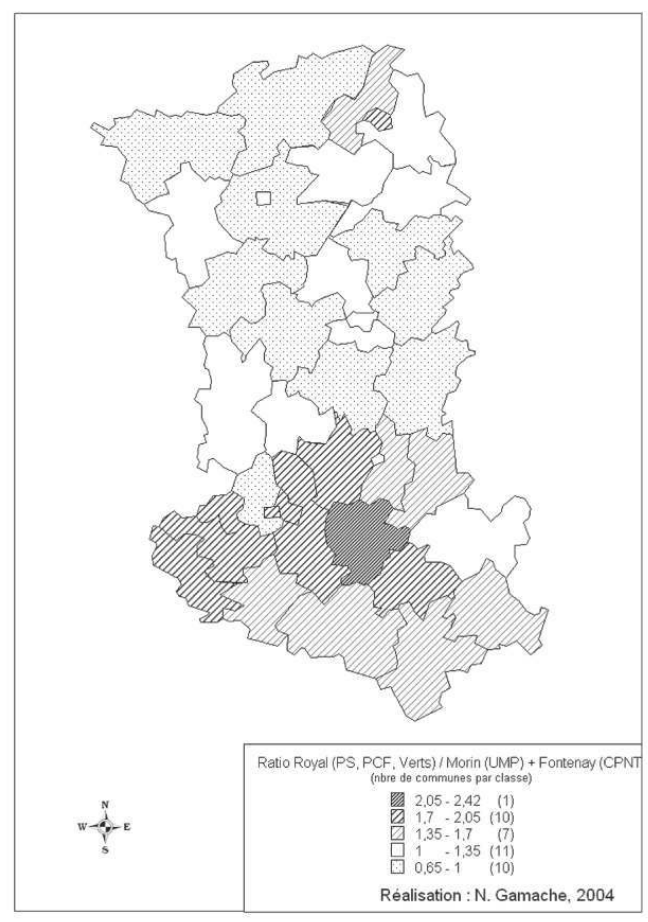

Le tableau 1 traduit en terme d'indices (Bussi et Badariotti, 2004) ces évolutions : l'indice de concentration nous informe sur la position des électorats et l'évolution de ces indices sur les tendances électorales.

L'examen de l'évolution des indices de concentration apporte une connaissance approfondie des territoires dans la durée, autrement que par les données brutes des taux de chaque candidat. En tout état de cause, les permanences, au moins sur les deux échéances analysées, ressortent clairement si l'on examine le resserrement des écarts types de ces indices. 
Tableau 1 : Indices de Concentration (IC) (élection présidentielle de 2002 et régionales de 2004) ${ }^{1,2}$ - (voir Bussi et al., 2004)

Concentration rating (IC) (2002 presidential election and 2004 regional election)

\begin{tabular}{|c|c|c|c|c|c|c|c|c|c|c|c|}
\hline Cantonsindiced & $\begin{array}{l}\text { IC gauche } \\
2002 \\
\text { (A) }\end{array}$ & $\begin{array}{l}\text { IC gauche } \\
2004 \\
\left(A^{\prime}\right)\end{array}$ & $\begin{array}{l}\text { Évolution IC } \\
\text { gauche } \\
\text { (Ev) }\end{array}$ & $\begin{array}{l}\text { Indice } \\
\text { gauche } \\
\text { (B) }\end{array}$ & $\begin{array}{l}\text { IC droite } \\
2002 \\
\text { (A) }\end{array}$ & $\begin{array}{l}\text { IC droite } \\
2004 \\
\left(A^{\prime}\right) \\
\end{array}$ & $\begin{array}{l}\text { Évolution } \\
\text { IC droite } \\
\text { (Ev) }\end{array}$ & $\begin{array}{l}\text { Indice } \\
\text { droite } \\
\text { (B) }\end{array}$ & $\begin{array}{l}\text { Exprimés } \\
2002\end{array}$ & $\begin{array}{l}\text { Exprimés } \\
2004\end{array}$ & $\begin{array}{l}\text { Évolution } \\
\text { des exprimés } \\
\text { (C) }\end{array}$ \\
\hline Bressuire & 88,67 & 85,91 & 96,89 & 82,13 & 112,43 & 126,56 & 112,57 & 95,42 & 16301 & 19230 & 117,97 \\
\hline Cerizay & 86,97 & 95,05 & 109,29 & 116,83 & 114,65 & 110,3 & 96,21 & 102,84 & 7426 & 6947 & 93,55 \\
\hline Mauléon & 60,64 & 69,19 & 114,10 & 134,71 & 132,38 & 148,05 & 111,84 & 132,04 & 8980 & 7606 & 84,70 \\
\hline Saint-Varent & 79,87 & 86,20 & 107,93 & 119,86 & 113,83 & 115,55 & 101,51 & 112,74 & 3052 & 2748 & 90,04 \\
\hline Thouars 1 & 96,41 & 98,71 & 102,39 & 111,67 & 104,18 & 100,21 & 96,19 & 104,91 & 5978 & 5481 & 91,69 \\
\hline Thouars 2 & 107,86 & 120,54 & 111,76 & 120,21 & 89,74 & 85,13 & 94,86 & 102,04 & 12096 & 11245 & 92,96 \\
\hline Airvault & 83,35 & 88,94 & 106,71 & 118,69 & 109,74 & 110,36 & 100,56 & 111,86 & 3060 & 2751 & 89,90 \\
\hline Champdeniers & 99,78 & 98,81 & 99,03 & 96,38 & 101,07 & 96,89 & 95,86 & 93,30 & 2949 & 3030 & 102,75 \\
\hline Coulonges & 89,15 & 93,41 & 104,78 & 112,45 & 101,81 & 104,86 & 103,00 & 110,54 & 5439 & 5068 & 93,18 \\
\hline Mazières & 82,08 & 86,77 & 105,71 & 115,28 & 118,27 & 121,33 & 102,59 & 111,87 & 3650 & 3347 & 91,70 \\
\hline Ménigoute & 81,43 & 83,50 & 102,54 & 100,47 & 110,84 & 121,27 & 109,41 & 107,20 & 2662 & 2717 & 102,07 \\
\hline Moncoutant & 70,97 & 75,00 & 105,68 & 117,93 & 129,63 & 143,61 & 110,78 & 123,63 & 6255 & 5605 & 89,61 \\
\hline Parthenay & 105,51 & 95,92 & 90,91 & 95,93 & 99,79 & 108,20 & 108,43 & 114,42 & 16446 & 15585 & 94,76 \\
\hline Secondigny & 72,36 & 79,45 & 109,80 & 118,51 & 125,88 & 131,49 & 104,46 & 112,74 & 4042 & 3745 & 92,65 \\
\hline Saint-Loup & 75,69 & 84,72 & 111,93 & 123,43 & 114,88 & 117,45 & 102,24 & 112,74 & 2393 & 2170 & 90,68 \\
\hline Thénezay & 68,83 & 79,14 & 114,98 & 123,58 & 119,16 & 129,98 & 109,08 & 117,24 & 2515 & 2340 & 93,04 \\
\hline Beauroir-sur-Niort & 89,56 & 103,64 & 115,72 & 108,01 & 105,04 & 91,73 & 87,33 & 81,51 & 2324 & 2490 & 107,14 \\
\hline Brioux-sur-Boutonne & 85,43 & 100,54 & 117,69 & 128,47 & 111,96 & 97,08 & 86,71 & 94,65 & 3705 & 3394 & 91,61 \\
\hline Celles-sur-Belle & 111,96 & 119,23 & 106,49 & 110,24 & 83,48 & 68,67 & 82,26 & 85,15 & 5471 & 5285 & 96,60 \\
\hline Chef-Boutonne & 91,63 & 101,49 & 110,76 & 122,31 & 106,71 & 90,92 & 85,20 & 94,08 & 3464 & 3137 & 90,56 \\
\hline Frontenay-Rohan-Rohan & 105,18 & 109,05 & 103,68 & 108,87 & 93,17 & 82,60 & 88,66 & 93,09 & 5389 & 5132 & 95,23 \\
\hline La Mothe-Saint-Heray & 94,92 & 104,84 & 110,45 & 107,66 & 100,58 & 88,88 & 88,37 & 86,13 & 2853 & 2927 & 102,59 \\
\hline Lezay & 94,94 & 96,84 & 102,00 & 102,16 & 101,29 & 100,99 & 99,70 & 99,86 & 3240 & 3235 & 99,85 \\
\hline Mauzé-sur-1e-Mignon & 98,42 & 104,97 & 106,66 & 109,27 & 100,05 & 83,03 & 82,99 & 85,02 & 3382 & 3301 & 97,60 \\
\hline Melle & 105,04 & 109,86 & 104,59 & 107,28 & 88,48 & 82,18 & 92,88 & 95,27 & 4947 & 4823 & 97,49 \\
\hline Saint-Maixent 1 & 106,64 & 108,89 & 102,11 & 107,89 & 86,76 & 83,17 & 95,86 & 101,28 & 7696 & 7284 & 94,65 \\
\hline Saint-Maizent 2 & 87,41 & 98,36 & 112,53 & 115,38 & 98,37 & 98,28 & 99,91 & 102,45 & 4603 & 4489 & 97,52 \\
\hline Sauzé-Vaussais & 85,78 & 99,31 & 115,77 & 120,04 & 101,18 & 92,81 & 91,73 & 95,11 & 3264 & 3148 & 96,45 \\
\hline Niort ouest & 117,57 & 108,45 & 92,24 & 100,41 & 85,99 & 84,94 & 98,78 & 107,52 & 11915 & 10946 & 91,87 \\
\hline Prahecq & 110,01 & 111,51 & 101,36 & 108,12 & 87,69 & 81,39 & 92,82 & 99,00 & 7107 & 6663 & 93,75 \\
\hline Niort ville & 124,34 & 109,06 & 87,71 & 95,29 & 84,07 & 85,60 & 101,82 & 110,62 & 26152 & 24072 & 92,05 \\
\hline Argenton-Château & 65,99 & 75,05 & 113,73 & 130,98 & 130,00 & 137,05 & 105,42 & 121,41 & 5512 & 4786 & 86,83 \\
\hline Moyennes & 91,39 & 96,32 & 106,18 & 112,20 & 105,10 & 103,77 & 98,13 & 103,68 & & & \\
\hline écarts types & 15,47 & 13,09 & 7,37 & 11,40 & 13,84 & 20,48 & 8,62 & 12,00 & & & \\
\hline
\end{tabular}

1. - Calcul de l'indice de concentration (Ic) : Ic $=(S(x) y / \Sigma S(x) \Sigma y) /\left(\Sigma e(y) / \Sigma e \sum y\right)$. Où : $x$ : un candidat ou parti ; y : un canton ; $\mathrm{S}$ : le score ; $\mathrm{E}$ : les exprimés. C'est-à-dire, l'indice de concentration est égal au score d'un candidat sur un canton divisé par l'ensemble des scores de ce candidat sur l'ensemble de tous les cantons, le tout divisé par les exprimés sur ce canton sur l'ensemble des exprimés de l'ensemble des cantons.

2. Calcul de l'indice de «tendance » (B) : I = (Ev) / (C) ; c'est-à-dire l'évolution de la concentration sur l'évolution des exprimés.

\section{Désalignement ou réalignement politique : les territoires porteurs de nouvelles expressions?}

Le coefficient de corrélation de Pearson ne montre pas de corrélation spatiale entre les votes protestataires (extrême droite et extrême gauche) et les différentes catégories socio-professionnelles (tableau 2). Cela ne signifie pas qu'il n'y ait pas de territorialité de ces votes. Le Front National par exemple se concentre sur toute la frange est du département (fig.3). Les relations au territoire sont là encore marquées par les caractéristiques sociologiques de ces territoires : les votes FN sont plus corrélés avec des populations plus âgées, à l'inverse des votes d'extrême gauche, touchant davantage les territoires d'implantation des cadres et professions intellectuelles, les espaces périurbains, plus jeunes et mobiles. 
Tableau 2 : Matrice des variables sociales et socio-professionnelles et votes (Coefficients de corrélation de Pearson)

En gras, valeurs significatives (hors diagonale) au seuil alpha $=0,050$ (test bilatéral). Matrix of social and socio-professional variables and votes (Pearson correlation coefficient)

\begin{tabular}{|c|c|c|c|c|c|c|c|c|c|}
\hline & \multicolumn{3}{|c|}{$2^{e}$ tour des élections régionales 2004} & \multicolumn{4}{|c|}{$1^{\text {er }}$ tour des élections présidentielles 2002} & \multirow[b]{2}{*}{$\begin{array}{l}\text { Indice de } \\
\text { jeunesse } \\
\text { électorale }\end{array}$} & \multirow[b]{2}{*}{$\begin{array}{l}\text { Taux de } \\
\text { mobilites } \\
\text { intercom. }\end{array}$} \\
\hline & $\begin{array}{c}\text { Vote Morin } \\
\text { (UMP) }\end{array}$ & $\begin{array}{c}\text { Vote Royal } \\
\text { (PS) }\end{array}$ & $\begin{array}{c}\text { Vote Char- } \\
\text { bonneau } \\
\text { (FN) }\end{array}$ & $\begin{array}{l}\text { Vote } \\
\text { droite }\end{array}$ & $\begin{array}{l}\text { Vote } \\
\text { gauche }\end{array}$ & $\begin{array}{l}\text { Vote } \\
\text { extrême } \\
\text { droite }\end{array}$ & $\begin{array}{l}\text { Vote } \\
\text { extrême } \\
\text { gauche }\end{array}$ & & \\
\hline $\begin{array}{l}\text { Taux de mobilites } \\
\text { intercommunales }\end{array}$ & $-0,526$ & 0,622 & $-0,040$ & $-0,388$ & 0,368 & $-0,007$ & 0,305 & 0,262 & 1,000 \\
\hline $\begin{array}{l}\text { Indice de jeunesse } \\
\text { électorale }\end{array}$ & $-0,290$ & 0,338 & $-0,519$ & 0,007 & 0,004 & $-0,122$ & 0,100 & 1,000 & 0,262 \\
\hline $\begin{array}{l}\text { Taux de proprié- } \\
\text { taires de leur } \\
\text { résidence }\end{array}$ & $-0,024$ & $-0,134$ & 0,325 & $-0,039$ & $-0,039$ & 0,334 & $-0,119$ & 0,088 & $-0,153$ \\
\hline $\begin{array}{l}\text { Taux d'actifs } \\
\text { agriculteurs }\end{array}$ & 0,587 & $-0,685$ & 0,392 & 0,318 & $-0,392$ & 0,276 & $-0,288$ & $-0,648$ & $-0,467$ \\
\hline $\begin{array}{l}\text { Taux d'actifs } \\
\text { artisans, commer- } \\
\text { çants, chefs d'entr. }\end{array}$ & 0,316 & $-0,386$ & 0.394 & 0,187 & $-0,119$ & 0,071 & $-0,387$ & $-0,699$ & $-0,307$ \\
\hline $\begin{array}{l}\text { Taux d'actifs } \\
\text { cadres et prof. } \\
\text { intellectuelles sup }\end{array}$ & $-0,603$ & 0,660 & $-0,319$ & $-0,298$ & 0,292 & $-0,157$ & 0,375 & 0,644 & 0,504 \\
\hline $\begin{array}{l}\text { Taux d'actifs prof. } \\
\text { intermediaires }\end{array}$ & $-0,592$ & 0,634 & $-0,415$ & $-0,282$ & 0,310 & $-0,208$ & 0,323 & 0,739 & 0.495 \\
\hline $\begin{array}{l}\text { Taux d'actifs } \\
\text { employés }\end{array}$ & $-0,615$ & 0,708 & $-0,089$ & $-0,263$ & 0,256 & $-0,063$ & 0,255 & 0,293 & 0,742 \\
\hline $\begin{array}{l}\text { Taux d'actifs } \\
\text { ouvriers }\end{array}$ & 0,491 & $-0,510$ & 0,046 & 0,188 & $-0,154$ & 0,000 & $-0,215$ & $-0,231$ & $-0,599$ \\
\hline
\end{tabular}

Si l'on suit l'explication (Bussi et Ravenel, 2001) du désalignement possédant/travailleur (pour le vote d'extrême gauche), centre/périphérie (pour l'extrême droite), rural/urbain (pour les votes « écolos » du type Verts ou CPNT), c'est-à-dire tous ceux issus des votes hors clivage traditionnel droite/gauche (désalignement État/Église), les Deux-Sèvres correspondent à l'image nationale. Les distinctions physico-culturelles s'estompent cette fois-ci et la cartographie électorale de ces désalignements dessinent de nouvelles territorialités (fig. 6). J.-R. Colle (1946) le notait déjà en 1946 pour la Gâtine où depuis la révolution, la ville de Parthenay est de gauche et la campagne de droite. Mais au vu des tendances relevées précédemment sur les évolutions des indices de concentration, l'expression d'un désalignement ne semble pas significative de l'évolution actuelle en Deux-Sèvres. Nous sommes ici en marge d'un réalignement politique qui mettrait à mal la bipolarité droite/gauche. 
Figure 6 : Territoires des nouvelles expressions : l'exemple des votes « écolos » (Verts et CPNT) au 1 er tour de l'élection Présidentielle 2002 en Deux-Sèvres

Territories of new voices: the example of ecologist vote (Verts and CPNT) at the 1st round of the 2002 Presidential election in Deux-Sèvres

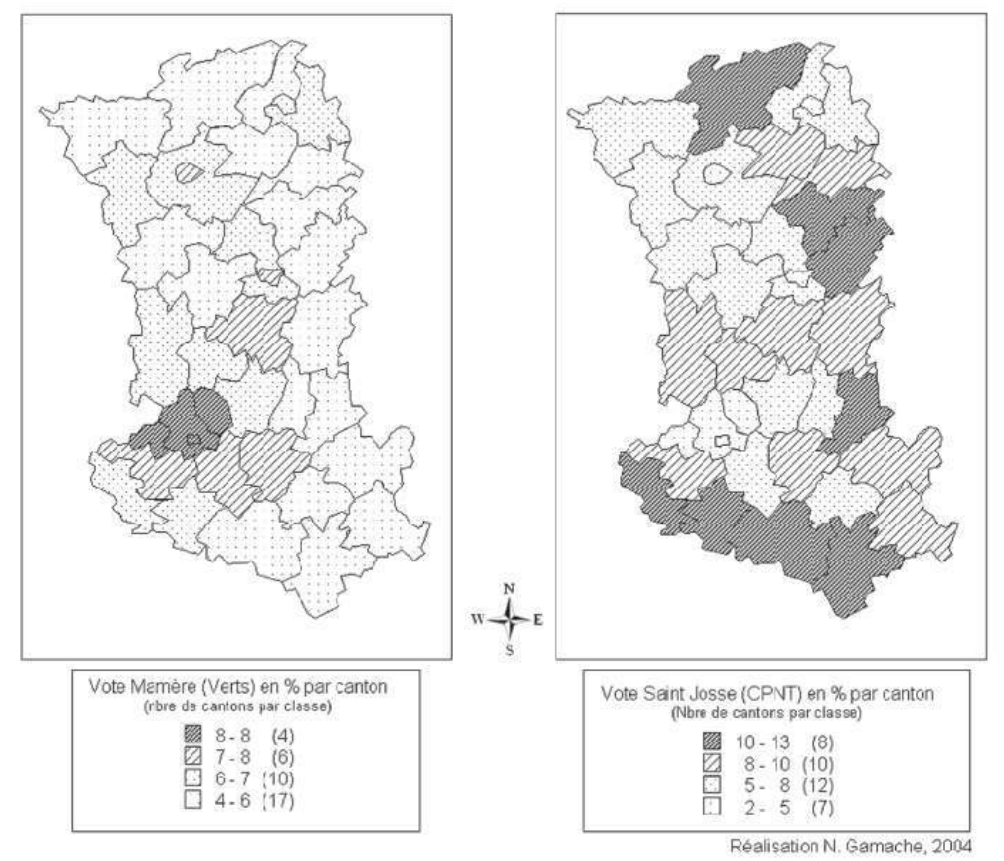

\section{Évolution électorale}

\section{Analyse selon trois champs exploratoires sur les Deux-Sèvres}

Par association spatiale, il est possible de démontrer l'existence d'un lien entre deux phénomènes. Cette méthode s'énonce par la « corrélation écologique » mais aboutit très souvent à "l'erreur écologique» (Robinson, 1950). En effet, interpréter une correspondance spatiale se révèle hasardeux comme nous l'évoquions plus haut. Partant de cette limite méthodologique, même si par ailleurs certains indicateurs statistiques permettent la comparaison entre cartes ou entre données, nous utiliserons ici trois éléments. La démonstration a pour but premier d'interroger des critères fortement liés aux territoires et mesurables quantitativement. Ces éléments portent sur des données relatives aux traits socio-démographiques des territoires. S'ils correspondent à des mesures issues des recensements par exemple et sont de nature contemporaine, il est à souligner une dynamique dans le temps assez constante quant à leur répartition dans l'espace : la figure 7 par exemple montre un taux d'agriculteurs plus élevé en nord DeuxSèvres qu'au sud. 
Figure 7 : Évolution de la part des agriculteurs dans la population active en Deux-Sèvres de 1982 à 1999 par canton

Evolution of the farmers'contribution in the working population of Deux-Sèvres from 1982 to 1999 by district

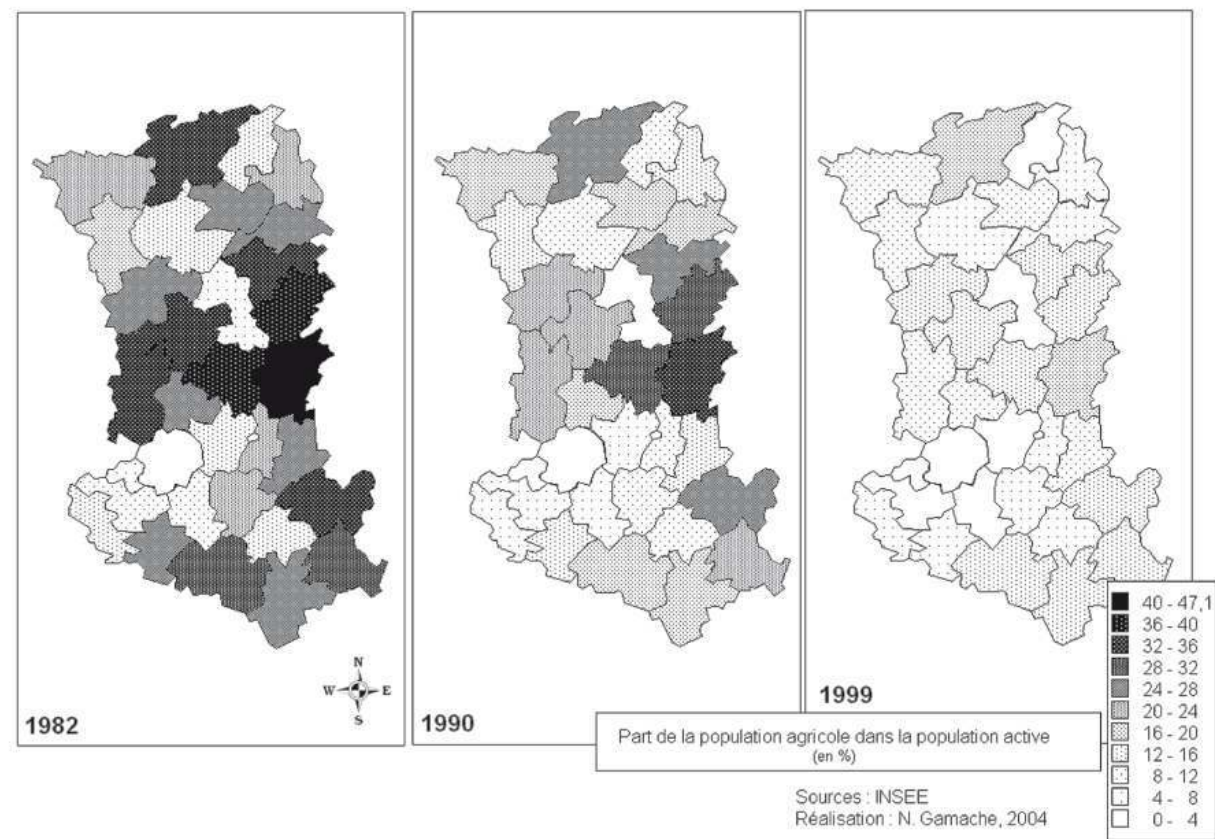

Si on assiste à une réduction des écarts essentiellement par le fait d'une baisse forte du nombre d'exploitants, les différenciations spatiales demeurent. D'autres exemples illustrent ces permanences territoriales dans la constance de différenciation spatiale: l'indice de jeunesse est toujours plus élevé dans le sud Deux-Sèvres qu'au nord par exemple.

\section{Vote et mobilités résidentielles}

Les mobilités résidentielles intercommunales entre les recensements de 1990 et 1999 (fig. 8) nous fournissent un matériau pour l'étude du rapport qu'entretiennent les votes au territoire. Les cantons à plus fortes mobilités sont essentiellement péri-urbains : couronne de Niort, moindrement de Thouars et Parthenay (Bressuire échappe à la statistique par le fait de sa fusion par le passé avec les petites communes "rurales », périurbaines aujourd'hui). Le contraste est d'autant plus fort avec les cantons ruraux et surtout ceux du nord Deux-Sèvres à mobilités très faibles. Deux possibilités s'offraient dans la correspondance entre ces mobilités et le sens du vote : soit par comparaison de cartes via la correspondance spatiale entre indices de concentration des votes et indice de répartition des mobilités, soit par calcul statistique de corrélation. 


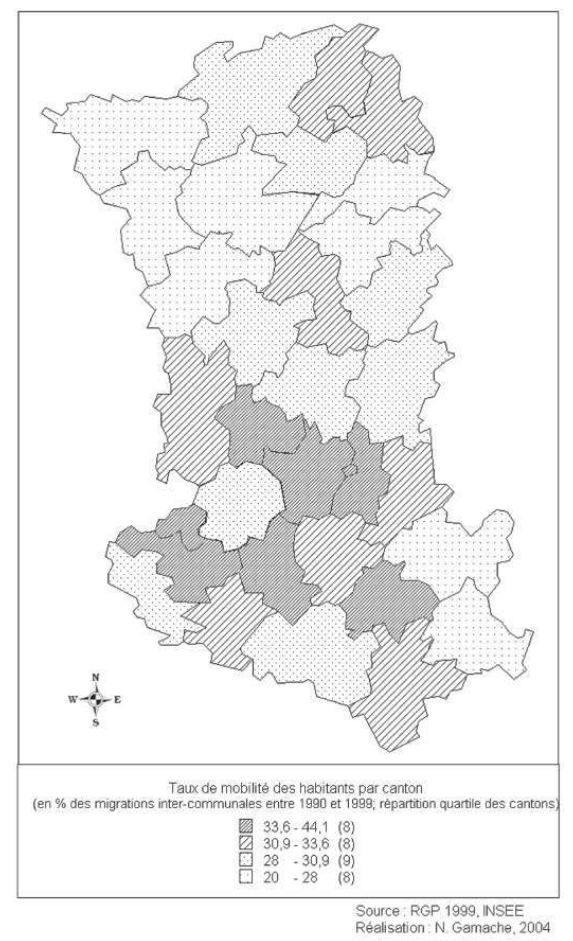

La seconde méthode permet de constater que le vote Morin (ou de droite, car ces résultats sont similaires sur les autres élections) est corrélé négativement $(-0,526)$ avec les mobilités et inversement pour le vote de gauche. Élément "perturbateur » dans le calcul de corrélation, Saint-Maixent-l'École, ville de l'École nationale des Sous-Officiers d'Active. Le taux de mobilité résidentiel élevé correspond au «turn over » des cadres de l'École et du personnel d'encadrement. Mais ce taux de mobilité élevé perturbe le modèle dans le sens où le vote à droite est bien plus élevé que pour les autres cantons, plus proche d'ailleurs de la droite d'ajustement. De là à dire que les militaires votent à droite, il n'y a qu'un pas qui est d'autant plus facile à franchir que la population Saint Maixentaise non militaire contient de nombreux cadres à la retraite ou de personnes issues du milieu. Un second point porte sur la position des plus fortes mobilités en sud Deux-Sèvres. On retrouve là une plus forte proportion d'employés, en Mellois par exemple et de logements sociaux.

\section{Vote et indice de « jeunesse électorale »}

31 L'âge étant un facteur de différenciation du vote, il est justifié d'examiner le lien entre géographie cantonale du vote et structure par âge de la population des cantons. «L'indice de jeunesse électorale " équivaut au rapport inter-génération sur les classes de population en âge de voter, soit le nombre de personnes en âge de voter âgées de moins de 60 ans divisé par le nombre de personnes âgées de plus de 60 ans. L'âge est un facteur de différenciation du vote, même s'il est arbitraire de fixer une limite à 60 ans. Ici, le rapport est à l'image de cette représentation des différenciations du vote national: les cantons avec les plus faibles indices de jeunesse votent davantage à droite que les autres. Cependant, le taux de corrélation est trop faible pour avaliser toute hypothèse de 
relation. Plusieurs singularités nous obligent à redoubler de prudence: St Maixent encore, par le fait d'une population plus jeune avec la présence de l'École militaire, abaisse à nouveau le taux de corrélation. Certains cantons du Bocage Bressuirais (comme Mauléon), ont un indice de jeunesse élevé mais votent inversement à la tendance catégorielle.

Les critères sociaux pris séparément sont donc limités dans leur utilisation possibles, surtout associés à une tentative de répartition spatiale. On retrouve en général des phénomènes liés dans l'espace physique de même que dans l'espace social: un canton agricole aura un taux de mobilité résidentiel faible, un indice de jeunesse également faible et votera davantage à droite. Inversement, une petite ville telle que Melle, avec davantage de logements sociaux, donc plus de mobilités résidentielles, aura un indice de jeunesse plus élevé, un taux de propriétaires plus faible et votera davantage à gauche.

\section{Catégories Socio-Professionnelles (CSP) et vote}

Le coefficient de corrélation de Pearson est utile pour traiter d'une géographie similaire ou opposée entre deux phénomènes. Dans le tableau 2 , ce coefficient se rapprochant de +1 indique une superposition des géographies entre deux phénomènes. Une corrélation nulle (coefficient de corrélation de Pearson proche de zéro) traduit probablement une indépendance des deux phénomènes envisagés, surtout si des régressions multiples confirment cette absence de lien supposée. Un fort coefficient négatif traduit des configurations spatiales opposées. À ce titre, les travaux de H. Le Bras (1995) fournissent des exemples intéressants. Parmi les enseignements possibles à tirer, il est à noter une certaine radicalisation des territorialités du vote. Le clivage territorial entre votes de droite et votes de gauche s'accentue dans sa corrélation avec le profil socio-professionnel de ces territoires, que ce soit l'un ou l'autre camp qui ait emporté les élections par ailleurs, même largement.

Les votes de droite sur les cantons à fort taux d'agriculteurs marquent l'électorat de référence dans les cantons de Gâtine et du bocage. Mais le poids de cette catégorie tend à diminuer et s'uniformiser sur l'ensemble départemental. La figure 7 montre un tassement du taux d'agriculteurs là où ils étaient déjà le moins nombreux en 1982, une diminution encore plus accélérée est enregistrée entre 1982 et 1999 dans ceux où ils étaient davantage présents dans la population active. L'écart type de la proportion d'agriculteurs dans les cantons des Deux-Sèvres est ainsi passé de 11,21 en 1982 à 8,85 en 1990 et 5,12 en 2000. Cette uniformisation des différenciations de catégories sociales au sein du département (tendance en tout cas pour cette catégorie sociale, mais largement diffuse aussi pour les autres avec par exemple une déconcentration des cadres ou des employés des milieux urbains vers le périurbain, voire rural) interroge donc sur un point de vue politiste. Elle donne l'obligation pour la droite d'un renouvellement de l'électorat au sein des milieux à fort taux d'agriculteurs ou de personnes âgées en adaptant le discours aux nouvelles catégories sociales à conquérir. C'est ce que M. Bussi (1993) appelle notamment «les mutations internes des courants». Une seconde question émerge, sur la relation de la répartition territoriale du vote en fonction des caractéristiques sociologiques des territoires. Va t-on premièrement vers une uniformisation des cantons d'un point de vue référence socio-professionnelle et si oui cela engendre t-il une uniformisation du vote?

À l'instar de la catégorie des agriculteurs, il n'est pas évident que les autres CSP suivent le même mouvement d'uniformisation (vers le bas). Certains cantons restent marqués par 
une forte présence d'ouvriers ou d'employés. La forte proportion d'ouvriers dans certains cantons n'implique pas nécessairement un vote de gauche ou d'extrême gauche comme traditionnellement, ni front national comme sur la tendance nationale (voir également l'article «L'après 28 mars/Les régions » dans Libération du 3 avril 2004 sur la question et sur les motivations aux changements du vote). Le canton de Cerizay par exemple connaît un réalignement du vote ouvrier vers le système droite-gauche, même s'il passe de l'un à l'autre.

Le bilan de l'analyse statistique nous montre que les différentes entités (nord/sud) du département des Deux-Sèvres gardent en elles une double tendance : d'une part les votes dépendent pour partie des conditions sociologiques de ces territoires, d'autre part ces critères sociologiques ne sont pas suffisants pour expliquer les différences de vote au sein de chaque groupe social et au sein de chacun des territoires. L'aspect sociologique explique les différentiations territoriales mais reste insuffisant pour rendre compte des permanences du vote de ces territoires.

\section{Étude de cas locale : le canton de Ménigoute}

37 M. Bussi (1993) insiste sur le fait d'une analyse électorale incomplète si elle se situe à l'échelle départementale, qui ne peut restituer toute l'étendue des diversités de comportements que seule l'analyse inter-régionale permet. C'est notamment le cas par la recherche explicative économique, où l'expression politique relevant des catégories professionnelles des habitants de bassins d'emplois (comme les ouvriers pour les bassins industriels, ou les mineurs etc.). Mais pour l'étude des permanences, l'échelle locale paraît pertinente, car à l'instar de l'analyse des facteurs économiques précités, les rapports sociaux et inter-personnels, voire individuels, n'ont plus la même portée qu'à l'échelle globale. Si un vote exprime une parenté à une sensibilité politique dans l'analyse à petite échelle, l'importance des relations inter-personnelles avec les élus notamment, du rapport au territoire et de la distance de l'échelon décisionnel pour lequel on vote, l'échelle locale recèle à ce titre des informations qu'il n'est possible de saisir qu'en s'intéressant à l'espace politique vécu.

\section{Les élections pour le conseil général de 2004 en Ménigoutais}

Dans la partie bocage des Deux-Sèvres, en Gâtine, le Ménigoutais est un canton du rural "profond » en déclin démographique, avec une recomposition de la population ( $48 \%$ des actifs étaient agriculteurs en 1982, moins de $20 \%$ aujourd'hui) et un vieillissement très accentué (Surault, 1988).

L'ancien Président du conseil général des Deux-Sèvres, conseiller général sortant, André Dulait (majorité départementale de droite), a vu son canton devenir le théâtre de remises en question de la continuité politique. Cela interroge sur ce territoire et les présumées permanences dont il serait porteur.

40 Aux élections cantonales 2004, 7 candidats étaient en lice pour prendre la place libérée par André Dulait : un candidat UMP, Philippe Albert, Maire de Vausseroux; un candidat étiqueté DVD par la Préfecture, se définissant lui-même "souverainiste", Maire de Fomperron, Serge Boutet; un conseiller régional sortant, classé DVG par la Préfecture, rangé à la majorité UMP au conseil régional, maire de Vasles, Gilles Parnaudeau ; une sans 
étiquette, Martine Grasset, rangée DVD, soutenue (au moins officieusement) par le conseiller sortant, habitante de Ménigoute; deux candidats « parachutés » (PCF et FN); et enfin un SE, identifié DVG par la préfecture, ne s'inscrivant pas dans la majorité départementale de droite dans sa profession de foi, conseiller municipal et communautaire de Vasles, mais résidant à Vausseroux, assureur de son métier, JeanCharles Pied. C'est ce dernier qui est élu au second tour, face à S. Boutet et G. Parnaudeau.

\section{Le local pour exprimer les permanences territoriales du vote}

La figure 9 montre la répartition des voies au premier tour. Comme nous l'avons dit précédemment, le canton est à chaque élection de droite, avec des distinctions entre communes : au nord de la Vonne (cours d'eau séparant le canton nord-sud), foyer plus catholique (Saint-Martin-du-Fouilloux et Vasles notamment) où les questions liées à l'école privée sont toujours d'actualité et vivement discutées, on vote davantage à droite. Seule commune à gauche à chaque élection, Coutières.

Figure $9: 1$ er tour des élections cantonales en Ménigoutais en 2004 1st round of the 2004 District election in Ménigoutais

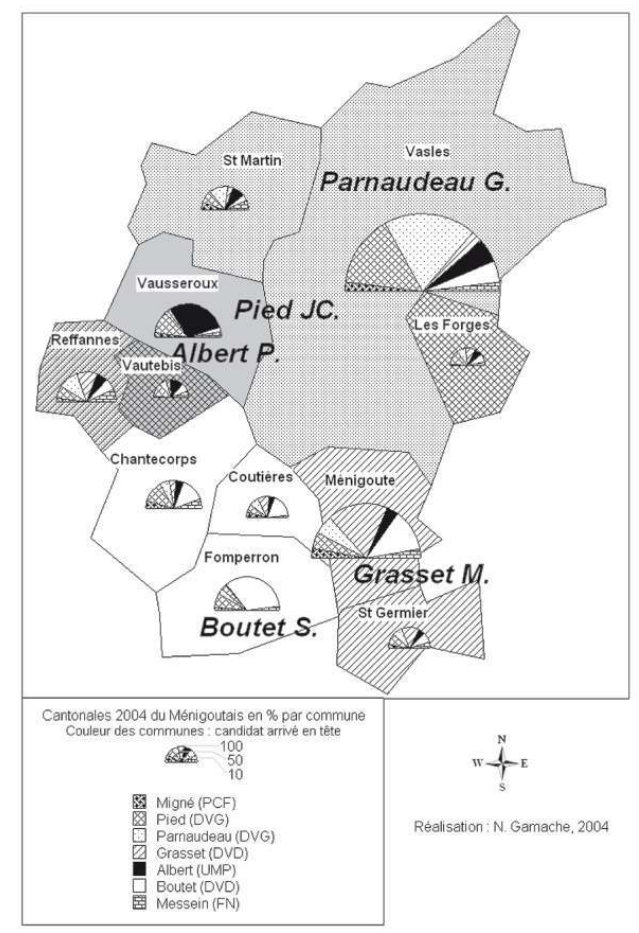

Ce qui est à souligner à travers l'étude cantonale du vote, à cette échelle et surtout pour une élection locale, le vote exprime moins les tendances politiques que les jeux d'influences liées à la position géographique des prétendants. On retrouve ainsi l'électorat de chacun des candidats autour de leur foyer, de résidence ou de mandat local. Si le territoire n'est pas ici porteur du vote mais exprime la proximité territoriale des candidats, il demeure essentiel dans la compréhension des permanences d'expression identitaire, politiques ensuite. Nous aborderons sous deux aspects les facteurs caractérisant la relation du vote et du territoire, ceux contribuant aux permanences et ceux à leur remise en cause. 

l'attention de certains candidats, notamment sur la question de projets et sur la question
financière. Réels ou non, les problèmes de la Communauté de communes projetés sur la scène électorale auront détourné l'attention de l'élection en jeu. Néanmoins, c'est un problème de représentation du territoire et de la manière dont chacun des protagonistes l'envisage. Derrière ces enjeux se profilent aussi celui des personnes. Entre ceux désireux (même s'ils ne l'avouent pas ainsi) d'accéder à une fonction élective "notable », et ceux mis en scène pour un parti ou d'autres personnes, les territoires sont aussi l'enjeu d'intérêts de personnes. Dans le temps, l'impact est de façonner l'idée d'un territoire autour d'une personne: André Dulait personnifie ainsi le Ménigoutais pour une large part.

48 Enfin, le contexte des élections, régional et national, influence le vote: ici, un vote sanction s'est exprimé envers la majorité départementale et nationale : le candidat UMP subit un échec dès le premier tour et la candidate officieusement soutenue par André Dulait échoue aussi dès ce premier tour. Le conseiller régional sortant fait les frais de son vote pour Jean-Pierre Raffarin au conseil régional.

Finalement, le vainqueur bénéficie du vote sanction des autres candidats, de son expérience communautaire et de son mandat électif de conseiller municipal, de son espace d'influence locale, de ses connaissances inter-personnelles liées à sa profession.

Dans un canton traditionnellement de droite, où chacun (ou presque) des candidats au poste de conseiller général prône le changement, mais dans la continuité (sic!), le candidat élu, qui se plaçait hors de la majorité sans se rapprocher de l'opposition, a finalement rejoint dans ses premiers votes, le parti pris de la continuité départementale 
(vote blanc pour la présidence du conseil général et vote du budget primitif). Cet exemple indique la forte identité d'un territoire et la difficulté pour ses acteurs politiques de se détacher d'une certaine continuité politique. On ne s'affranchit pas facilement, surtout en milieu rural et terre de tradition, du poids des héritages. Peut-être les tractations des élus et les relations inter-personnelles des élus entre eux et aux citoyens influencent-elles également leur démarche dont ils sont le moteur mais aussi l'enjeu...

Pour finir, ce qui fait continuité ou rupture, c'est le rapport des forces endogènes et des forces exogènes au territoire. Le vécu des relations politiques au territoire conditionne les valeurs dont il est porteur, qu'il s'agisse de stabilité du champ politique en son sein ou de renouveler le débat par de nouvelles donnes.

\section{Conclusion}

Religieux, certes, car influencé par les conditions physiques du milieu, si l'on considère les rapports sociaux et les rapports au monde dont elles sont en partie la source; social, incontestablement: l'expression de la condition sociale s'opère pleinement; le vote trouve ses logiques explicatives dans tous ces facteurs sans leur être complètement asservi. Les contres exemples ne manquent pas pour défaire un modèle électoral reposant sur un ou plusieurs de ces critères. Mais une variable demeure incontournable dans la compréhension des permanences électorales, comme nous l'aura montré l'exemple deuxSèvrien : aux dernières élections, tout comme le relataient déjà R. Bobin en 1926 et J.-R. Colle en 1946, les tendances politiques ont une forte prégnance territoriale. La survivance des comportements électoraux au-delà des transformations sociologiques du territoire (mobilités, géographiques ou sociales), par la mémoire collective notamment, tient à ce que le propre du territoire soit de durer. L'étude de l'histoire sociale sur le temps long met en relief ce fait. Le territoire, expression polymorphe d'espaces de vie, circonscrit aussi l'espace politique. On peut se demander si ce dernier est instrumentalisé par les acteurs. Il serait alors plus qu'un support ou un repère, mais un vecteur du pouvoir.

\section{BIBLIOGRAPHIE}

BADARIOTTI (D.), 2004. - « De la comparaison des cartes électorales », revue électronique Cybergéo, $\mathrm{n}^{\circ} 267,17 \mathrm{p}$.

BERTRAND (J.-R.), MULLER (C.), 1999. - Religions et territoires, Paris, L'Harmattan, 304 p.

BOBIN (R.), 1926. - La Gâtine, étude de géographie, Niort, Impr. A. Chiron, 221 p.

BUSSI (M.), BADARIOTTI (D.), 2004. - Pour une nouvelle géographie du politique, Paris, Anthropos, 301 p. BUSSI (M.), COLANGE (C.), GOSSET (J.-P.), FOURQUET (J.), 2004. - « Élections régionales 2004, deuxième tour : plongée socio-électorale sous la vague rose », revue électronique Cybergéo, rubrique «Points chauds ». 
BUSSI (M.), RAVENEL (L.), 2001. - «Écologistes des villes, écologistes des champs. Analyse spatiale du vote des Verts et du CPNT », revue électronique Cybergéo, $\mathrm{n}^{\circ} 205$.

BUSSI (M.), FREIRE-DIAZ (S.), 2002. - « Les nouvelles spatialités des comportements électoraux français, l'exemple des élections présidentielles 1981-1995 », revue électronique Cybergéo, rubrique Points chauds

BUSSI (M.), 1993. - « Les trois systèmes explicatifs de l'Ouest politique », Norois, t. 40, n 158, p. 193-215

COLLE (J.-R.), 1946. - En Gâtine, Niort, éditions du Vieux-Marais, 136 p.

GUILLOREL (H.), 1991. - «L'espace breton et le mouvant », dans LÉVY (J.) (dir.), Géographies du politique, Paris, Presses de la FNSP, $100 \mathrm{p}$.

LACOSTE (Y.), 1986. - Géopolitiques des régions françaises, Paris, Fayard, t. 2, 1372 p.

LE BRAS (H.), 1995. - Les trois France, Paris, Odile Jacob, $2^{\mathrm{e}}$ édition, 464 p.

MERLE (L.), 1990. - La formation territoriale du département des Deux-Sèvres, Niort, Société historique et scientifique des Deux-Sèvres, $338 \mathrm{p}$.

RAVignan (F. de), Roux (P.), 1990. - L'Atlas de la France Verte, Paris, Jean-Pierre de Monza, 220 p.

ROBINSON (W. S.), 1950. - « Ecological correlation and the behaviour of individuals ", American

Sociological Review, XV, p. 351-357.

SCHEIBLING (J.), 1994. - Qu'est-ce que la géographie, Paris, Hachette, 199 p.

SIEGFRIED (A.), 1913. - Tableau politique de la France de l'Ouest, Paris, A. Colin, XXVIII, 536 p.

SURAUlT (P.), 1988. - « La diffusion du vieillissement dans les campagnes de Poitou-Charentes », Norois, t. $35, \mathrm{n}^{\circ} 140$, p. 443-452.

Vidal de La Blache (P.), 1994. - Tableau de la géographie de la France, Paris, La Table Ronde, 560 p.

\section{RÉSUMÉS}

Au-delà des « Raz de marée bleus » ou des «vagues roses » qui ont déferlé sur la France lors des élections ces dernières années, des permanences territoriales façonnent le paysage politique de certains départements. Sur un plan géologique, en Deux-Sèvres, le nord bocager se caractérise largement par son socle granitique. Profondément marquée par l'empreinte religieuse catholique, cette partie du département se rattache à une France de l'Ouest de droite. Plus généralement de champs ouverts, le sud du département repose sur une roche mère calcaire. Davantage athée voire influencée par le protestantisme, cette partie des Deux-Sèvres est teintée $\mathrm{du}$ rose politique du Sud-Ouest français. Si la géographie électorale recherche souvent ses systèmes explicatifs à l'échelle nationale dans des facteurs sociologiques et religieux, nous investissons ici d'autres champs d'analyse possibles : temps long, facteurs socio-démographiques et territoires de proximité. Le caractère territorial du vote sera ainsi mis en valeur.

Whether the right or the left wins elections, widely or not, deeply rooted territorial influences imprint the French political scene. In Deux-Sèvres, the north of the woodlands on granite, clearly marked by religion, is attached to the political France of the West (which is conservative). The South of the "department" on limestone of openfields, more atheistic, even influenced by Protestantism, is supporting the left such as the Southwest of France. If the electoral geography 
inquires into its explanatory systems in sociological and religious factors, on a national scale, we'll look into other possible fields of analysis: long time, socio-demographic factors and territories of proximity. The territorial aspect of the vote will be thus emphasized.

INDEX

Index géographique : Deux-Sèvres

Mots-clés : géographie électorale, paysage, territoire

Keywords : electoral geography, landscape, territory

\section{AUTEUR}

NICOLAS GAMACHE

Université de Poitiers

Faculté de l'Aménagement - Université de Montréal

EA 2252-Identité et Connaissance des Territoires et Environnements en Mutation - CNRS

nicolasgamache79@aol.com 